\title{
Vignettes in the history of chemistry. 1. What is the origin of the Gibbs-Helmholtz equation?
}

\author{
William B. Jensen ${ }^{1}$
}

Received: 16 November 2015 / Accepted: 10 December 2015/Published online: 27 January 2016

(C) Springer International Publishing Switzerland 2016

\begin{abstract}
This column is intended to address questions students and teachers may have about the historical origins of a symbol, name, concept or experimental procedure found in their textbook. If there is a specific subject you would like to see addressed in the series, feel free to e-mail your queries to Dr. Jensen at the above address. This particular installment traces the historical origins of the well-known GibbsHelmholtz equation and casts doubt on the role of Gibbs in its formulation. Target audience: chemistry courses at all levels depending on the subject being discussed.
\end{abstract}

Keywords History of chemistry · Physical chemistry · Thermodynamics · Gibbs-Helmholtz equation

The first problem in attempting to answer the question in the above title is the ambiguous way in which the term "Gibbs-Helmholtz equation" is used in the thermodynamic literature to describe at least a dozen different equations, albeit all of them derivable from one another [1]. Thus, in their simplest form, equations bearing this name may involve either the Helmholtz free-energy function $(A)$ :

$(\partial A / \partial T)_{V}=-S$

the Gibbs free-energy function $(G)$ :

$(\partial G / \partial T)_{P}=-S$

or the electrochemical cell potential $(E)$ :

$(\partial E / \partial T)_{P}=\Delta S / v_{\mathrm{e}} F$

William B. Jensen

Jensenwb@ucmail.uc.edu

1 Department of Chemistry, University of Cincinnati, Cincinnati, OH 45221-0172, USA where $F$ is Faraday's constant and $v_{\mathrm{e}}$ is the stoichiometric coefficient for the moles of electrons transferred in the balanced half-cell reactions.

The name may also be applied to a variety of more complex equations derived from the above relationships, as illustrated below for the specific case of the Gibbs freeenergy function:

$$
\begin{aligned}
& \Delta H=\Delta G-T(\partial \Delta G / \partial T)_{P} \\
& \Delta G=\Delta H+T(\partial \Delta G / \partial T)_{P} \\
& \partial(\Delta G / T) / \partial T)_{P}=-\Delta H / T^{2} \\
& \partial(\Delta G / T) / \partial(1 / T)_{P}=\Delta H
\end{aligned}
$$

Historically, versions of Eqs. 1 and 3 were first derived by the German physicist, Hermann von Helmholtz (Fig. 1), in 1882, as well as the Helmholtz free-energy and electrochemical cell potential equivalents of Eq. 4 [2]. ${ }^{1}$

$$
\begin{aligned}
& \Delta U=\Delta A-T(\partial \Delta A / \partial T)_{V} \\
& \Delta U=-v_{\mathrm{e}} F\left[E-T(\partial E / \partial T)_{V}\right]
\end{aligned}
$$

Trying to determine when and where the American physicist, Josiah Willard Gibbs (Fig. 2), first derived an equivalent equation or equations has, however, proved to be much more difficult and involves several false leads. Thus, for example, Partington, in his 1913 treatise on chemical thermodynamics, states [3]:

There is a very important equation relating to the electromotive forces of reversible cells which was deduced independently by J. Willard Gibbs (1875) and H. von Helmholtz (1882), and is usually called the Gibbs-Helmholtz equation.

\footnotetext{
${ }^{1}$ Not unexpectedly, the symbolism used by Helmholtz, as well as some sign conventions, differs from the modern variants used here.
} 


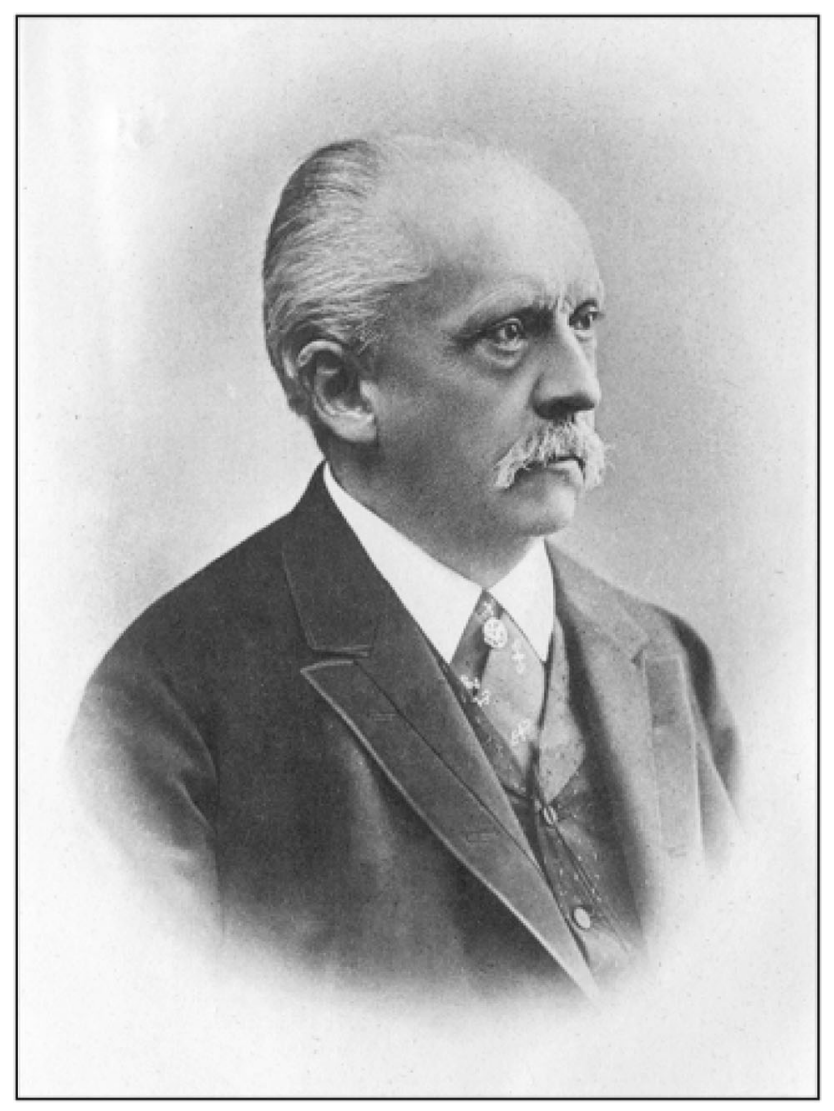

Fig. 1 Hermann von Helmholtz (1821-1894)

However, an examination of Gibbs' epic 1875 paper, "On the Equilibrium of Heterogeneous Substances" [4], fails to reveal any such equations, and 31 years later Partington would correct his earlier statement in Volume 4 of his massive History of Chemistry, where he admitted that the Gibbs-Helmholtz equation "was never given or used by Gibbs" [5].

But if this is the case, how was Partington misled in 1913? The answer seems to lie in a passing comment in L. P. Wheeler's 1951 biography of Gibbs, where we read [6]: Another physicist who, according to the testimony of Professor Michael Pupin, must have become acquainted with Gibbs' work in the mid-eighties was Hermann von Helmholtz. In 1882, the latter had published a derivation of an equation giving the rate of change of the electromotive force with temperature for a reversible electrolytic cell which generally became known as the Gibbs-Helmholtz. equation after it was pointed out that it was implicit and more readily derivable from Gibbs' work of some 4 or 5 years previous.

There are three things to note in this quote: first, the unsubstantiated suggestion that Helmholtz had gotten the idea for his equation from the earlier work of Gibbs; second, the admission that the equation in question was only

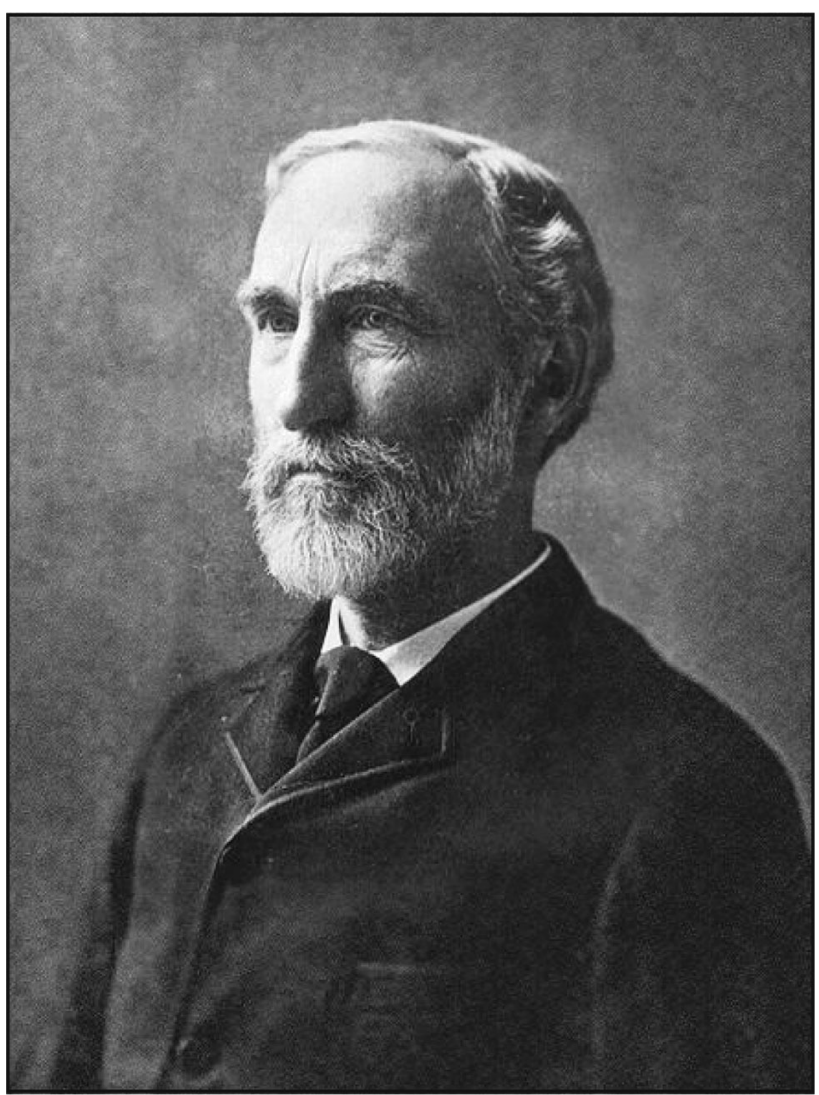

Fig. 2 Josiah Willard Gibbs (1839-1903)

"implicit" in that work; and third, Wheeler's failure to tell us who it was that first called attention to this fact.

As it turns out that someone was probably none other than Gibbs himself in a letter to Sir Oliver Lodge published in the 1888 volume of the Report of the Meetings of the British Association for the Advancement of Science. Here, after reviewing the equations he had given in 1875 for the potential of an electrochemical cell at constant temperature, he notes that [7]: ${ }^{2}$

It is very easy to show that these results are in complete accordance with Helmholtz's differential equation. We have only to differentiate (with respect to the temperature) the value which we have found for the electromotive force.

After six subsequent substitutions and rearrangements, Gibbs finally arrives at his version of Eq. $3,{ }^{3}$ albeit 6 years

\footnotetext{
${ }^{2}$ As in the case of Helmholtz, there are differences in both Gibbs' choice of symbols and sign conventions versus our modern usages. In addition, Gibbs, unlike Helmholtz, completely ignores the issue of units and unit conversions.

3 The discrepancy between the timing in Partington versus Wheeler is due to the fact that the difference is 7 years if one uses the date 1875 , which is when the first installment of Gibbs' memoir was published, but only 4 years if one takes into account that the section dealing with electrochemistry did not appear until 1878.
} 
after Helmholtz rather than 7 years before him, as originally implied by Partington and Wheeler.

Thus, we find that Partington's conclusion in 1964 was no more correct than his statement of 1913. But does the derivation of an already known equation 6 years after the fact really entitled one to equal billing? This question is even further complicated by Partington's further claim in his history that relationships equivalent to Eq. 4 had already been derived by both Horstmann in 1872 and by Lord Kelvin in 1857, though I have been unable to verify this claim $[5,8] .{ }^{4}$

All of these difficulties are a reflection of the pitfalls that underlie the widespread practice of eponymy or the naming of equations, laws, elements, compounds, apparatus, etc. after famous scientists. For a variety of reasons, these names are almost invariably historically misleading and it would save much gnashing of teeth on the part of historians if this practice was discontinued. This, of course, is unlikely to happen since canonization in this fashion is probably the unspoken fantasy of every practicing scientist.

Acknowledgments The author would like to acknowledge Paul Mathias of Fluor Corporation, Aliso Viejo, CA 92698 for submitting this question.

\section{References}

1. James AM (1976) A dictionary of thermodynamics. Wiley, New York, pp 116-117

2. Helmholtz H (1882) Die Thermodynamik chemischer Vorgänge. Sitzber Kgl Preuss Akad Wissensch 22-39. Planck M (ed) (1902) Abhandlungen zur Thermodynamik von Hermann Helmholtz. Engelmann, Leipzig 17-36

3. Partington JR (1913) A text-book of thermodynamics. Constable, London: 456

4. Gibbs JW (1875-1878) On the equilibrium of heterogeneous substances. Trans Conn Acad 3: 108-248, 343-524. Reprinted in Bumstead HA, van Name, RG (eds) (1906) The scientific papers of J. Willard Gibbs. Vol. 1, Longmans Green \& Co, London: 55-353

5. Partington JR (1964) A history of chemistry. Vol. 4, Macmillan, London: 615

6. Wheeler LP (1951) Josiah Willard Gibbs: the history of a great mind. Yale, New Haven: 97

7. Gibbs JW (1888) Letter to the secretary of the electrolysis committee of the BAAS. Report Brit Assoc Adv Sci: 343-346. Reprinted in Bumstead HA, van Name, RG (eds) (1906) The scientific papers of J Willard Gibbs. Vol. 1, Longmans Green \& Co, London: 408-412

8. Jensen WB (2009) August Horstmann and the origins of chemical thermodynamics. Bull Hist Chem 34:83-91

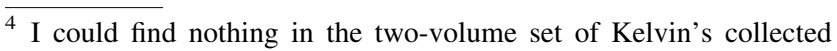
papers or in Horstmann's publications on thermodynamics, for more on Horstmann and his lesser known contributions, see [8].
} 